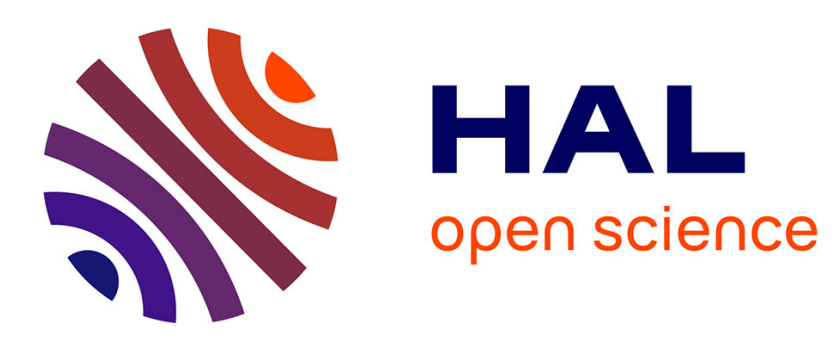

\title{
Transition frequency of transport ac losses in high temperature superconducting coated conductors
}

\author{
Pengbo Zhou, Guangtong Ma, Loic Queval
}

\section{To cite this version:}

Pengbo Zhou, Guangtong Ma, Loic Queval. Transition frequency of transport ac losses in high temperature superconducting coated conductors. Journal of Applied Physics, 2019, 126 (6), pp.3901. 10.1063/1.5094727 . hal-02505363

\section{HAL Id: hal-02505363 \\ https://hal.science/hal-02505363}

Submitted on 17 Jul 2020

HAL is a multi-disciplinary open access archive for the deposit and dissemination of scientific research documents, whether they are published or not. The documents may come from teaching and research institutions in France or abroad, or from public or private research centers.
L'archive ouverte pluridisciplinaire $\mathbf{H A L}$, est destinée au dépôt et à la diffusion de documents scientifiques de niveau recherche, publiés ou non, émanant des établissements d'enseignement et de recherche français ou étrangers, des laboratoires publics ou privés. 


\section{Transition frequency of transport ac losses in high temperature superconducting coated conductors}

Cite as: J. Appl. Phys. 126, 063901 (2019); https://doi.org/10.1063/1.5094727

Submitted: 05 March 2019 . Accepted: 15 July 2019 . Published Online: 08 August 2019

Pengbo Zhou (D), Guangtong Ma, and Loïc Quéval (iD)

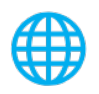




\title{
Transition frequency of transport ac losses in high temperature superconducting coated conductors
}

Cite as: J. Appl. Phys. 126, 063901 (2019); doi: 10.1063/1.5094727

Submitted: 5 March 2019 . Accepted: 15 July 2019.

Published Online: 8 August 2019

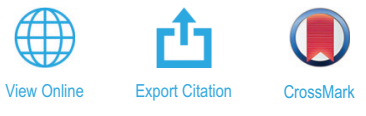

Pengbo Zhou, ' (D) Guangtong Ma, ${ }^{1, a)}$ and Loïc Quéval ${ }^{2}$ (D)

\section{AFFILIATIONS}

${ }^{1}$ Applied Superconductivity Laboratory, State Key Laboratory of Traction Power, Southwest Jiaotong University, Chengdu, Sichuan 610031, China

${ }^{2}$ Group of Electrical Engineering_Paris (GeePs), CNRS UMR 8507, CentraleSupélec, UPSud, Sorbonne University, Gif-sur-Yvette 91192, France

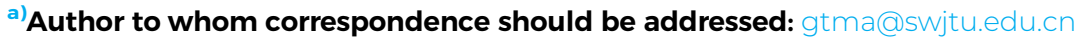

\begin{abstract}
Experimental data reveal that the classical description of transport ac losses in high-temperature superconducting (HTS)-coated conductors (CCs), based on investigations at low frequencies, is incomplete in some aspects when transport currents in the kilohertz range are considered. More specifically, above a certain "transition frequency," the ac losses per cycle no longer increase with the frequency as the theory predicts. Using a finite element model to allow for loss separation, we find that this phenomenon is caused by a combination of several factors that appear only above the transition frequency: the hysteresis and ferromagnetic losses per cycle are no longer independent of the frequency, while the eddy current losses per cycle no longer increase proportionally to the frequency. Based on a circuit model, we propose that the physical reason for this is that when the frequency increases, part of the supercurrent starts migrating into the metallic path. We argue that the current in the metallic path is not an eddy current but a transport current inductively coupled to the superconducting current. Finally, we discuss the relationship between the magnetic material magnetization, the critical current, and the transport current frequency. This study provides explicit insights into the frequency-dependent transport ac losses of HTS CCs in a broad frequency band, which is valuable for the design and optimization of HTS CC-based power devices.
\end{abstract}

Published under license by AIP Publishing. https://doi.org/10.1063/1.5094727

\section{INTRODUCTION}

There have been significant improvements in high-temperature superconducting (HTS)-coated conductors (CCs) in terms of conductor quality and length availability. This makes them promising candidates for a wide range of electrical applications, ${ }^{1}$ such as transmission cables, ${ }^{2,3}$ machinery, ${ }^{4,5}$ transformers, ${ }^{6,7}$ flux pumps, ${ }^{8-10}$ and wireless power transfer systems. ${ }^{1-16}$ However, when they carry ac currents, HTS conductors are subject to power losses. These losses, called transport ac losses, can cause problems with regard to cryogenic stability and overall efficiency. Understanding and management of such losses is, therefore, of paramount importance for practical applications of superconductors. ${ }^{17,18}$

An HTS CC is a composite made of superconducting material, magnetic material(s), and metallic material(s), each of which may make a non-negligible contribution to the ac losses, depending on the frequency, that must be accounted for. The widely accepted theoretical approach to this problem divides the ac losses of an HTS CC operating below its critical current into three categories: hysteresis losses caused by magnetic flux penetration and movement in the superconducting material; ferromagnetic losses caused by magnetic hysteresis in the magnetic material; and eddy current losses caused by currents induced by varying local magnetic fields in the metallic material. ${ }^{17,19}$ These losses exhibit various frequency dependences: the hysteresis and ferromagnetic losses per cycle are independent of the frequency, while the eddy current losses per cycle increase proportionally to the frequency. ${ }^{20-22}$ Thus, the ac losses per cycle of an HTS CC, being the sum of the above-mentioned contributions, should increase proportionally to the frequency.

Considerable efforts have been made to complete this theoretical description. At low frequencies, for example, experimental results indicated that the transport ac losses per cycle in an HTS CC actually first slightly decrease before increasing proportionally 
to the frequency. ${ }^{23,24}$ Further experimental and numerical results showed that this is because the hysteresis losses per cycle, which are dominant at very low frequencies, slightly decrease proportionally to the frequency. ${ }^{25-28}$ Furthermore, Thakur et al. ${ }^{28}$ included the impact of the frequency-dependent critical current in the Norris equation for the hysteresis losses of a current-carrying thin strip. Supported by experimental data, they demonstrated that the hysteresis losses per cycle in fact decrease with the frequency as $f^{-2 / n}$ ( $n$ being the exponent of the E-J power law) for ac currents well below the critical current and at low frequencies (maximum $400 \mathrm{~Hz}$ ).

At relatively higher frequencies, several studies have been carried out. The transport ac losses of HTS CCs with various magnetic substrates were investigated both experimentally and numerically up to $1 \mathrm{kHz}$. It was found that an enhancement of the substrate magnetism can increase both the hysteresis losses and the eddy current losses. ${ }^{21,29-34}$ In addition, Chen et al. ${ }^{35} \mathrm{cal}-$ culated analytically the transport ac losses of a superconducting core surrounded by a copper shell. They suggested that the current in the metallic material is not the commonly defined eddy current, without any experimental validation. In addition, a series of numerical and experimental investigations on the frequency-dependent ac losses of Bi2223-Ag-sheathed tapes were conducted up to $2.5 \mathrm{kHz}$. The results confirmed that the eddy current losses per cycle increase linearly with the frequency. ${ }^{36-39}$ More recently, this property has been further demonstrated by a comparative experimental study of the transport ac losses in HTS tapes with and without copper stabilizers up to $1 \mathrm{kHz}$ (and numerically up to $10 \mathrm{kHz}) .^{40}$

If the aforementioned experiments considered only frequencies under $2.5 \mathrm{kHz}$, this may be due to the difficulty of measuring accurately the transport ac losses of HTS CCs at high frequencies. ${ }^{35}$ In this study, we extend the measurement of the transport ac losses of a HTS CC with magnetic substrate up to $15 \mathrm{kHz}$. To improve the measurement precision, we use a spiral voltage loop arrangement. The results show that the theoretical description of the frequencydependent losses is incomplete, in particular as the frequency increases. To understand the underlying mechanism, we establish a finite element (FE) model taking the real dimensions of the HTS $\mathrm{CC}$ into account. This enables us to separately estimate the losses in each constituent of the HTS CC. Furthermore, to propose a reasonable explanation for the observations, we develop a circuit model. In the following, we designate the losses in the metal components as "metallic losses" rather than "eddy current losses" for reasons that will be detailed later.

This paper is structured as follows. In Sec. II, we present the experimental setup to measure the high-frequency transport ac losses of HTS CCs. In Sec. III, we detail the modeling and the finite element analysis. In Sec. IV, we compare the measured losses and the simulated ones. We discuss the findings with the help of a circuit model (described in the Appendix). Finally, in Sec. V, we summarize the key results of this work.

\section{EXPERIMENTAL SETUP}

The HTS CC used in this study is a commercially available product from American Superconductor. Figure 1 shows its SEM cross-sectional view. The CC is prepared using rolling-assisted
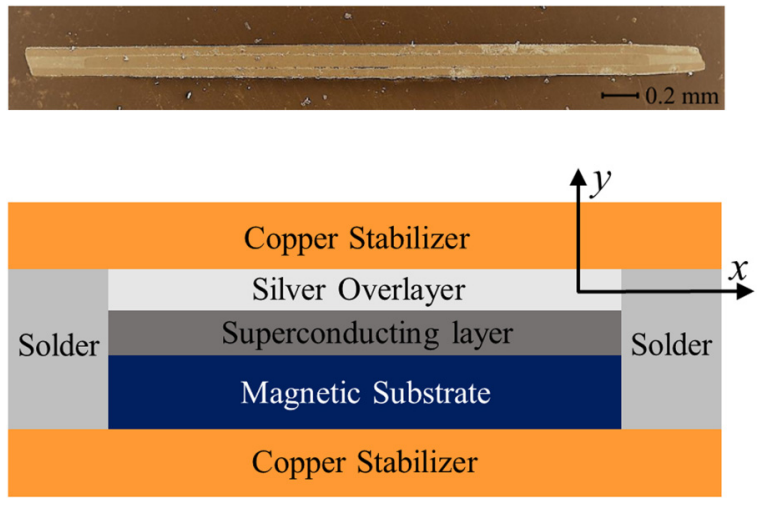

FIG. 1. RABiTS HTS CC: (top) SEM view of the cross section; (bottom) schematic of the cross section (not to scale). The superconducting layer is covered by a silver overlayer and deposited on a magnetic substrate. They are sandwiched by upper and lower copper stabilizers spliced by solder fillets. The thicknesses of each constituent from top to bottom are $50,3,1,75$, and $50 \mu \mathrm{m}$, respectively. The superconducting layer, silver overlayer, and magnetic substrate have equal widths of $4 \mathrm{~mm}$. The copper stabilizers have widths of $4.8 \mathrm{~mm}$, and the solder fillets have thicknesses of $79 \mu \mathrm{m}$ and widths of $0.4 \mathrm{~mm}$.

biaxially textured substrates (RABiTS). It is made up of five constituents: a superconducting layer, a silver overlayer, a Ni-5at\%W magnetic substrate, top and bottom copper stabilizers, and solder fillets.

We measured the self-field current-voltage of the HTS CC at $77 \mathrm{~K}$. A critical current of $108 \mathrm{~A}$ for a criterion of $1 \mu \mathrm{V} \mathrm{cm}$ was obtained, which is close to the value of $103 \mathrm{~A}$ given by the manufacturer.

We measured the self-field transport ac losses of the HTS CC at $77 \mathrm{~K}$ employing the lock-in amplifier technique. The frequency of the transport current was varied from $210 \mathrm{~Hz}$ to $15 \mathrm{kHz}$, well above the upper limit of previous works. If few works have investigated ac losses at such frequencies, this might be because of the difficulty of carrying out accurate high-frequency ac loss measurements. To be more specific, as the Norris theory points out, to correctly measure ac losses, the voltage measurement loop should link the magnetic flux from the center of the tape to a sufficiently large distance, ${ }^{41}$ at least three times greater than the tape half-width. ${ }^{42-44}$ Unfortunately, such a large voltage measurement loop would pick up, in addition to the resistive (in-phase) voltage, an inductive (out-of-phase) voltage that can be several orders of magnitude larger. ${ }^{45}$ This undesirable component is even more significant at high frequencies, since the inductive voltage is a linear function of the frequency. To address this problem, we employed a spiral voltage loop arrangement (Fig. 2), which reduces the measured out-of-phase voltage signal and the interference from the stray fields. ${ }^{46}$ We underline that we did not utilize any cancellation coil, which is otherwise universally employed in loss measurement, to compensate for the inductive voltage. The reason is that if the compensation voltage is not purely inductive, it will add some unwanted resistive voltage to the in-phase voltage. ${ }^{47}$ Besides, according to Tsukamoto et al., ${ }^{45}$ above $200 \mathrm{~Hz}$ the cancellation coil could cause non-negligible measurement errors. 


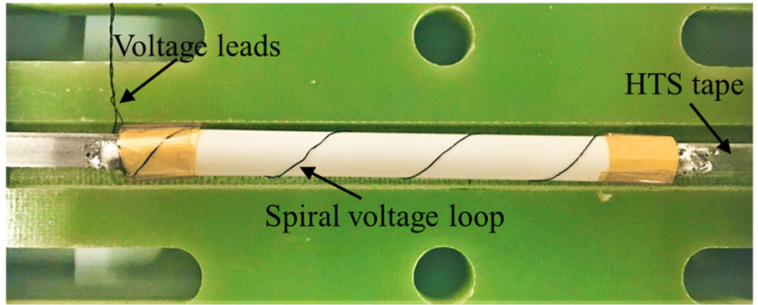

FIG. 2. Photograph of the spiral voltage loop arrangement for high-frequency transport ac loss measurements. To obtain accurate measurements, the voltage loop should be a spiral of constant radius and constant pitch. The number of turns of the spiral should be an integer. In this study, the voltage loop was wound on a cylindrical plastic tube with a constant pitch of $2 \mathrm{~cm}$, and the distance between the voltage taps was $8 \mathrm{~cm}$.

Figure 3 is a schematic diagram of the experimental setup. We utilized a digital lock-in amplifier (Stanford RS830) to generate the reference sine signal. The transport current was obtained by amplifying the reference signal with two parallel connected bipolar power supplies (Kikusui PBZ 20-20). The amplitude of the transport current was limited to $45 \mathrm{~A}$, corresponding to the maximum current of the power supply at hand. The voltage of the sample was measured by a spiral voltage loop that was connected to the lock-in amplifier. To synchronize the reference sine signal to the transport current, a Rogowskii coil coupled to the current lead was adopted. Meanwhile, restricted by the output current of our power supply, we connected the sample directly to the power supply without using any isolation transformer. With this configuration, the whole system has a common ground point. Accordingly, we used the differential floating input of the lock-in amplifier without grounding the sample. The ac losses per cycle per unit length $Q_{t}$ of the HTS CC are obtained as

$$
Q_{t}=\frac{I_{t, r m s} V_{r, r m s}}{f l},
$$

where $I_{t, r m s}$ is the root mean square (RMS) value of the transport current, $V_{r, r m s}$ is the RMS value of the sample resistive (in-phase)

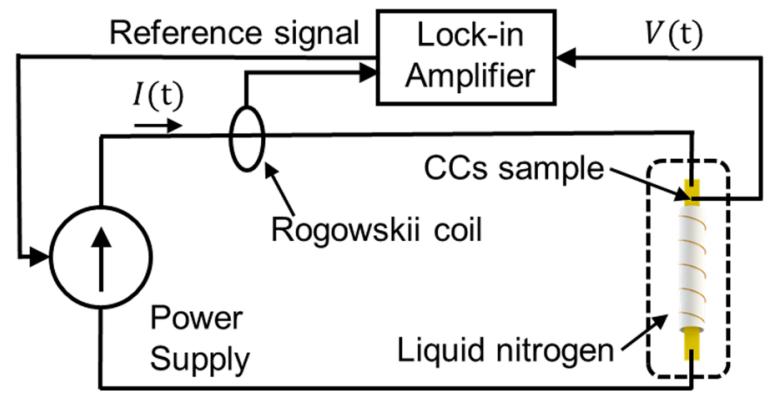

FIG. 3. Schematic of the experimental setup to measure high-frequency transport ac losses. voltage, $f$ is the frequency of the transport current, and $l$ is the distance between the two voltage taps.

\section{FINITE ELEMENT MODEL}

Experimentally, only the transport ac losses can be obtained. To allow for loss separation, a 2D $\mathbf{H}$-formulation finite element (FE) model was established. ${ }^{48}$ It takes the real dimensions of the HTS CC and the field-dependent properties of the magnetic substrate into account. ${ }^{49,50}$ The governing equations in the model consist of Faraday's law, Ampère's law, and the material constitutive law,

$$
\begin{gathered}
\nabla \times \mathbf{E}=-\mu_{0} \frac{\partial \mu_{r} \mathbf{H}}{\partial t}, \\
\nabla \times \mathbf{H}=\mathbf{J}, \\
\mathbf{E}=\rho \mathbf{J},
\end{gathered}
$$

where $\mu_{0}$ is the vacuum magnetic permeability, $\mu_{r}$ is the relative magnetic permeability, and $\rho$ is the material resistivity. Considering the translational symmetry of the HTS CCs, the current has only a $z$ component $\mathbf{J}=\left[0,0, J_{z}\right]$. Thus, the magnetic and electrical fields can be represented as $\mathbf{H}=\left[H_{x}, H_{y}, 0\right]$ and $\mathbf{E}=\left[0,0, E_{z}\right]$. Equations $(2 \mathrm{a})-(2 \mathrm{c})$ can then be rewritten as

$$
\begin{gathered}
\mu_{0} \frac{\partial \mu_{r} H_{x}}{\partial t}+\frac{\partial E_{z}}{\partial y}=0, \\
\mu_{0} \frac{\partial \mu_{r} H_{y}}{\partial t}-\frac{\partial E_{z}}{\partial x}=0, \\
J_{z}=\frac{\partial H_{y}}{\partial x}-\frac{\partial H_{x}}{\partial y}, \\
E_{z}=\rho J_{z} .
\end{gathered}
$$

Equations (3a) $-(3 d)$ are solved with the partial differential equation (PDE) module of the commercial software COMSOL. The superconductor resistivity $\rho_{s c}$ is modeled with an E-J power law combined with a Kim-like model to include the magnetic field dependence of the critical current, ${ }^{51,52}$

$$
\begin{gathered}
\rho_{s c}(\mathbf{J}, \mathbf{B})=\frac{E_{c}}{J_{c}(\mathbf{B})}\left|\frac{\mathbf{J}}{J_{c}(\mathbf{B})}\right|^{n-1}, \\
J_{c}(\mathbf{B})=\frac{J_{c 0}}{\left(1+\sqrt{k^{2} B_{\|}^{2}+B_{\perp}^{2}} / B_{0}\right)^{\alpha}} .
\end{gathered}
$$

Here, $E_{c}=1 \mu \mathrm{V} \mathrm{cm}^{-1}$ refers to the electric criterion for the critical current, $n=30$ is the power-law exponent, $B_{\|}$and $B_{\perp}$ are the parallel and perpendicular components of the magnetic flux density with respect to the surface of the tape, respectively. $B_{0}, k$, and $\alpha$ are material-related parameters with values of $0.0425,0.29515$, 
and 0.7 , respectively. ${ }^{51} J_{c 0}$ is the self-field critical current density derived from the measured self-field critical current $I_{\mathrm{c} 0}=108 \mathrm{~A}$. The resistivities of copper, silver, magnetic substrate, and solder at $77 \mathrm{~K}$ are $1.97,2.7,63$, and $31 \mathrm{n} \Omega \mathrm{m}$, respectively. ${ }^{53,54}$

The field-dependent relative magnetic permeability $\mu_{r}(\mathbf{H})$ of the $\mathrm{Ni}-\mathrm{W}$ substrate is defined by a function fitted from experiments, ${ }^{30,49,55,56}$

$$
\begin{aligned}
\mu_{r}(\mathbf{H})=\mu_{0}\{ & 1+30600\left[1-\exp \left(-\left(\frac{|\mathbf{H}|}{295}\right)^{2.5}\right)\right]|\mathbf{H}|^{-0.81} \\
& \left.+45 \exp \left(-\left(\frac{|\mathbf{H}|}{120}\right)^{2.5}\right)\right\},
\end{aligned}
$$

where $|\mathbf{H}|=\sqrt{H_{x}^{2}+H_{y}^{2}}$. The relative magnetic permeability is assumed to be 1 for the other materials. The transport current $I_{t}$ flowing in the HTS CC is imposed by an integral constraint on the current density over the HTS CC cross section $\Omega_{\mathrm{sc}}$,

$$
I_{t}(t)=I_{p} \sin (\omega t)=\int_{\Omega_{\mathrm{sc}}} J_{z}(t) d \Omega,
$$

where $I_{p}$ is the peak transport current and $\omega$ is the angular frequency. The hysteresis losses in the superconducting material and the metallic losses in the metallic material, per cycle per unit length, are calculated by integration of the power density over the cross section and over the second half of the first steady-state period,

$$
Q_{i}=2 \int_{T / 2}^{T} \int_{\Omega_{i}} E_{z} J_{z} d \Omega d t
$$

where $T$ is the period and $\Omega_{i}$ is the domain of interest. The ferromagnetic losses per cycle per unit length in the magnetic substrate are determined from the local maximum magnetic field $B_{m}$ as ${ }^{30,49,56}$

$$
\begin{gathered}
Q_{f e}=\int_{\Omega_{s}} q_{f e} d \Omega, \\
q_{f e}= \begin{cases}4611.4 B_{m}^{1.884}, & B_{m} \leq 0.164 T, \\
210\left\{1-\exp \left(-\left(6.5 B_{m}\right)^{4}\right)\right\}, & B_{m}>0.164 T,\end{cases}
\end{gathered}
$$

where $\Omega_{s}$ is the substrate domain.

\section{RESULTS AND DISCUSSION}

\section{A. Transition frequency of transport ac losses}

The measured ac losses per cycle of the sample as a function of the transport current frequency are plotted in Fig. 4. A feature revealed by the measurements is that there exists a "transition frequency" $f_{T}$ around $2 \mathrm{kHz}$ above which the theoretical description of the frequency-dependent losses is inaccurate. Specifically, below $f_{T}$, the ac losses per cycle increase proportionally to the frequency as expected. But over $f_{T}$, the ac losses per cycle decrease proportionally to the frequency, which is in conflict with the classical theoretical prediction.

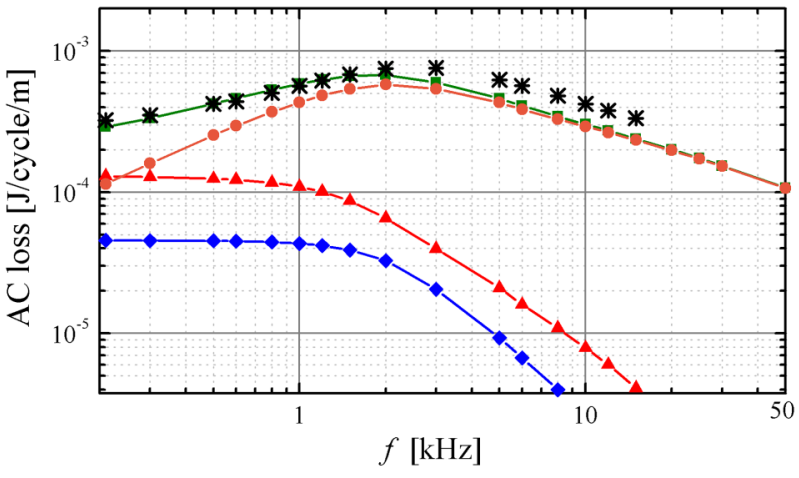

FIG. 4. Measured (asterisks) and FE-simulated (line with rectangles) transport ac losses per cycle per unit length as a function of the frequency with transport current $I_{p}=45 \mathrm{~A}$. The different loss components are also shown: hysteresis losses (line with triangles), ferromagnetic losses (line with diamonds), and metallic losses (line with circles).

\section{B. General features of the loss contributions}

To clarify the observation made above, the simulated ac losses have been added to Fig. 4. The simulation is able to reproduce the experimental results. The good agreement between measurements and simulations tends to confirm the correctness of the FE model, which can therefore be used to evaluate the losses separately in each domain.

The simulated loss contributions have been added to Fig. 4 . To improve readability, the hysteresis losses over $8 \mathrm{kHz}$ and the ferromagnetic losses over $12 \mathrm{kHz}$ are not shown, since their contributions to the ac losses are negligible. The following can be observed: (i) The ac losses per cycle are governed almost solely by the metallic losses, especially at high frequencies. This is in agreement with the conclusions of other groups. ${ }^{21,35,40}$ (ii) The metallic losses per cycle display an unexpected feature: they first increase up to $f_{T}$, then decrease. This indicates that the metallic losses are physically different from the so-called eddy current losses, which should be linearly proportional to the frequency. This is why we call these losses "metallic losses" instead of "eddy current losses." (iii) The hysteresis losses per cycle are nearly independent of the frequency below $f_{T}$. Above $f_{T}$, they decrease continuously, but with a faster trend than reported by previous work. ${ }^{28}$ (iv) The ferromagnetic losses per cycle are independent of the frequency below $f_{T}$. However, an unforeseen decay is observed at higher frequencies. (v) The ac losses, being the sum of the hysteresis losses, the ferromagnetic losses, and the metallic losses, increase proportionally to the frequency below $f_{T}$ and decrease proportionally to the frequency above $f_{T}$.

Comparing the magnitudes of the loss contributions, we can conclude that the observed frequency dependence of the transport ac losses is mainly due to the frequency transition of the metallic losses. In an attempt to clarify the underlying causes, a circuit model of the HTS CC with transport current was established (see the Appendix). Before applying this circuit model, its validity will be assessed by comparison with the FE model. Assuming that the transport current is always lower than the critical current, we neglect the 
superconducting resistance $R_{s c}$. Defining the complex transport current $\bar{I}_{t}=I_{t} e^{j \varphi_{t}}$, we obtain the complex metallic current $\bar{I}_{m}=I_{m} e^{j \varphi_{m}}$,

$$
\begin{gathered}
\bar{I}_{m}=\frac{\bar{V}-j \omega M \bar{I}_{s c}}{R_{m}+j \omega L_{m}}=\bar{I}_{t} \frac{j \omega\left(L_{s c}-M\right)}{R_{m}+j \omega\left(L_{s c}+L_{m}-2 M\right)}, \\
I_{m}(\omega)=I_{t} \frac{\omega\left(L_{s c}-M\right)}{\sqrt{R_{m}^{2}+\omega^{2}\left(L_{s c}+L_{m}-2 M\right)^{2}}}, \\
\varphi_{m}(\omega)=\varphi_{t}+\frac{\pi}{2}-\arctan \left(\frac{\omega\left(L_{s c}+L_{m}-2 M\right)}{R_{m}}\right) .
\end{gathered}
$$

Similarly, we obtain the complex supercurrent $\bar{I}_{s c}=I_{s c} e^{j \varphi_{s c}}$,

$$
\begin{aligned}
\bar{I}_{s c} & =\bar{I}_{t} \frac{R_{m}+j \omega\left(L_{m}-M\right)}{R_{m}+j \omega\left(L_{s c}+L_{m}-2 M\right)}, \\
I_{s c}(\omega)= & I_{t} \frac{\sqrt{R_{m}^{2}+\omega^{2}\left(L_{m}-M\right)^{2}}}{\sqrt{R_{m}^{2}+\omega^{2}\left(L_{s c}+L_{m}-2 M\right)^{2}}}, \\
\varphi_{s c}(\omega)= & \varphi_{t}+\arctan \left(\frac{\omega\left(L_{m}-M\right)}{R_{m}}\right) \\
& -\arctan \left(\frac{\omega\left(L_{s c}+L_{m}-2 M\right)}{R_{m}}\right) .
\end{aligned}
$$

At low frequencies $\left[\omega \ll R_{m} /\left(L_{s c}+L_{m}-2 M\right)\right]$, the metallic current increases proportionally to the frequency and has a phase shift of about $\pi / 2$ with respect to the phase of the transport current. ${ }^{21,31,35}$ When the frequency increases, part of the supercurrent starts migrating into the metallic path: the metallic current is no longer proportional to the frequency and has a phase inferior to $\pi / 2$. Eventually, at high frequencies $\left[\omega \gg R_{m} /\left(L_{s c}+L_{m}-2 M\right)\right]$, the amplitude of the metallic current tends to a constant value and is in phase with the total transport current. The trends deduced from the circuit model are confirmed by the FE model (see Fig. 5). We conclude that the circuit model seems to be able to qualitatively explain the observations. But its utilization for a quantitative analysis is limited owing to the difficulty of determining the resistance and inductance of a conductor with a nonuniform current distribution.

\section{Hysteresis losses}

In this subsection, we discuss the observed frequency dependence of the hysteresis losses per cycle. These are often considered to be independent of frequency, but we observed a decrease above the transition frequency $f_{T}$. Even when the frequency dependence of $I_{c}$ is introduced, ${ }^{28}$ the hysteresis losses should decrease as a function of $f^{-2 / n}$, which is much slower than the observed decrease. Consequently, other factors, more significant than the frequency dependence of $I_{c}$, must come into play. We argue here that the explanation can be found by combining the Norris equation ${ }^{41}$ and Eq. (11b). Norris gives the hysteresis losses per cycle in a thin strip of superconductor
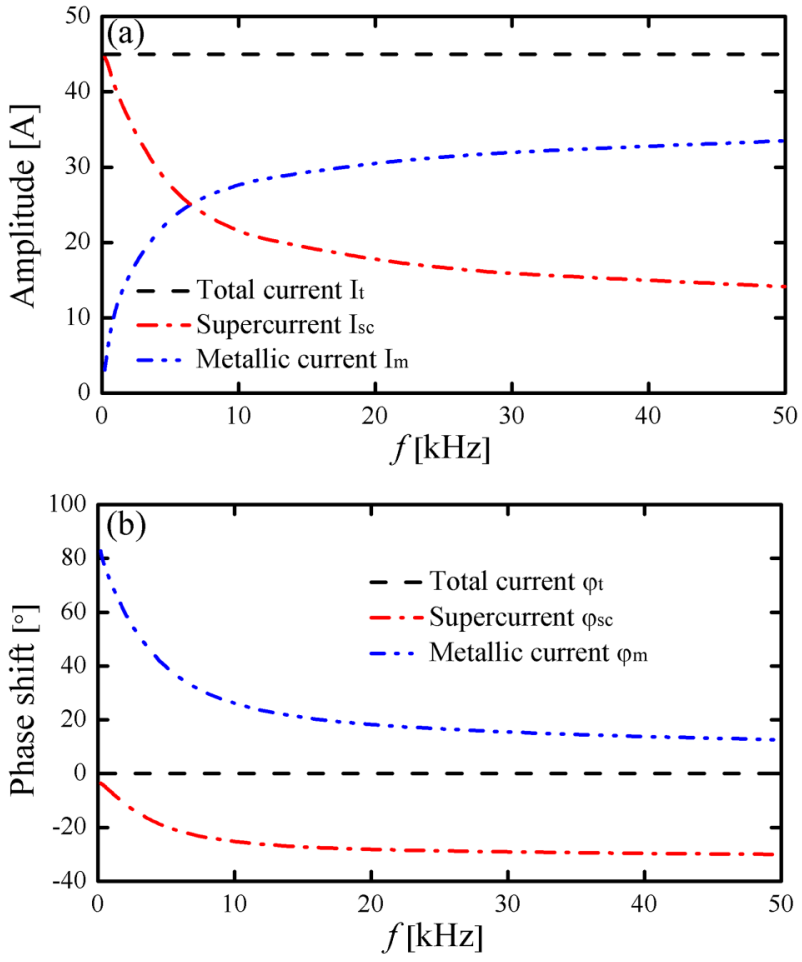

FIG. 5. FE-simulated amplitude (a) and phase (b) of the supercurrent and metallic current as functions of the frequency with transport current $I_{p}=45 \mathrm{~A}$. The phase of the transport current is the reference phase.

in the transport current case as

$$
\begin{aligned}
Q_{s c}\left(I_{\mathrm{sc}}\right)=\frac{\mu_{0} I_{c}^{2}}{\pi}[ & \left(1+\frac{I_{\mathrm{sc}}}{I_{c}}\right) \log \left(1+\frac{I_{\mathrm{sc}}}{I_{c}}\right) \\
& \left.+\left(1-\frac{I_{\mathrm{sc}}}{I_{c}}\right) \log \left(1-\frac{I_{\mathrm{sc}}}{I_{c}}\right)-\left(\frac{I_{s c}}{I_{c}}\right)^{2}\right],
\end{aligned}
$$

where $I_{c}$ is the critical current of the superconductor and $I_{s c}$ is the transport current. $Q_{s c}$ is a monotonically increasing function of $I_{\mathrm{sc}}$. Hence, when the supercurrent decreases with the frequency (Fig. 5), so do the hysteresis losses per cycle.

\section{Metallic losses}

In this subsection, we discuss the observed frequency dependence of the metallic losses per cycle. These are often considered to increase proportionally with the frequency, but we observed in this study a decrease above the transition frequency $f_{T}$. This can be explained by inserting Eq. (10b) into the expression for the metallic losses per cycle $Q_{m}$,

$$
Q_{m}=\frac{R_{m} I_{m}^{2}}{f}=2 \pi R_{m} I_{t}^{2} \frac{\omega\left(L_{s c}-M\right)^{2}}{R_{m}^{2}+\omega^{2}\left(L_{s c}+L_{m}-2 M\right)^{2}} .
$$


The derivative is then given by

$$
\frac{d Q_{m}}{d \omega}=2 \pi R_{m} I_{t}^{2}\left(L_{s c}-M\right)^{2} \frac{R_{m}^{2}-\omega^{2}\left(L_{s c}+L_{m}-2 M\right)^{2}}{\left[R_{m}^{2}+\omega^{2}\left(L_{s c}+L_{m}-2 M\right)^{2}\right]^{2}} .
$$

It can be seen that $Q_{m}$ reaches a maximum for $\omega_{T}=2 \pi f_{T}=R_{m} /\left(L_{s c}+L_{m}-2 M\right)$. This is qualitatively in agreement with the observed results. It is worth noting that we can conclude from Eq. (10a) that the current in the metallic constituent is not an induced eddy current as it is commonly regarded, but a transport current driven by the power supply, with a frequency dependence linked to the self-inductance of the metallic path $L_{m}$ and to the inductive coupling between the metallic path and the superconducting path $M$.

\section{E. Ferromagnetic losses}

In this subsection, we discuss the observed frequency dependence of the ferromagnetic losses per cycle. These are often considered to be independent of frequency, but we observed in this work a rapid decrease above the transition frequency $f_{T}$. To explain the trend, we argue here that the losses vary because of the variation of the local magnetic field. We assume that most of the metallic current flows in the lower copper stabilizer (see Sec. IV F). From Fig. 5, we have $\varphi_{m}-\varphi_{s c}<\pi / 2$, and thus the magnetic field of the HTS CC can be schematically plotted as shown in Fig. 6. This figure demonstrates that the field generated by the supercurrent always tends to oppose the field generated by the current flowing in the lower copper stabilizer. Besides, as shown in Sec. IV B, the current in the superconductor layer decays, while the current in the lower copper stabilizer increases with the frequency. As a result of these two facts, the local magnetic field decreases in the substrate when the frequency rises. This is confirmed by looking at the field distribution in the magnetic substrate obtained from the FE model (Fig. 7). As a consequence, the ferromagnetic losses, which depend on the peak magnetic field according to Eqs. (9a) and (9b), decrease when the frequency increases.

\section{F. Impacts of the magnetic substrate}

\section{Impact on hysteresis losses}

As reported in the literature, the presence of a magnetic substrate strongly increases the hysteresis losses of an HTS CC. ${ }^{57,58}$ This is because the substrate enhances the magnetic field in the

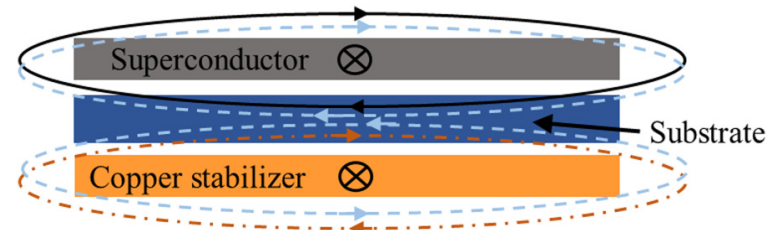

FIG. 6. Schematic of the HTS CC cross section and idealized magnetic field lines generated by the supercurrent (solid line), by the magnetized substrate (dashed line), and by the current in the lower copper stabilizer (dashed-dotted line).

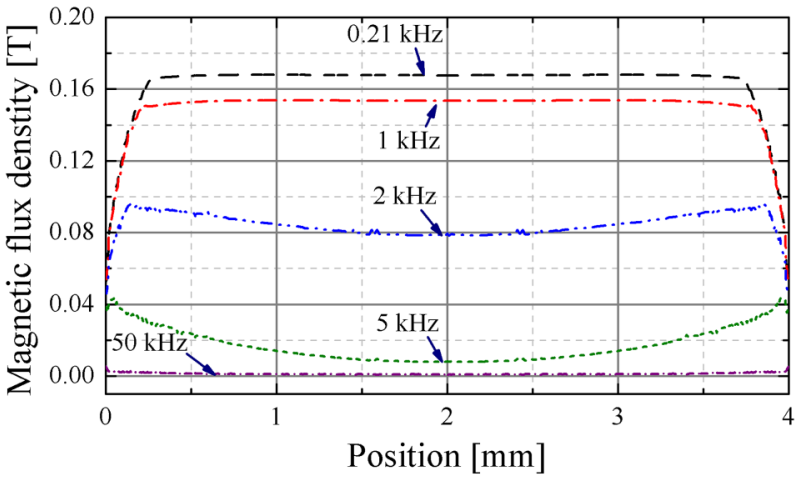

FIG. 7. FE-simulated magnetic flux density (norm) along the centerline of the substrate at the time instant when the transport current reaches its maximum. The horizontal axis varies from 0 to $4 \mathrm{~mm}$ (the width of the substrate) with reference to Fig. 1.

superconducting layer, lowering the critical current according to Eq. (5). But, as demonstrated in Sec. IV E, the magnetization of the substrate decreases with the frequency. Therefore, the critical current increases with the frequency in the presence of a magnetic substrate. This effect contributes to further reduce the hysteresis losses at high frequencies.

\section{Impact on metallic losses}

As the metallic losses become prominent at high frequencies, it is essential to understand their distribution. We plot the metallic loss contributions obtained with the FE model in Fig. 8. It can be seen that the metallic losses originate mainly from the copper stabilizers, especially from the lower one. This is in accordance with previous reports. ${ }^{29,31}$

According to Liu et al., ${ }^{21}$ the lower copper stabilizer has greater losses than the upper copper stabilizer because it has a stronger local magnetic flux density. We argue here, to the contrary, that the real

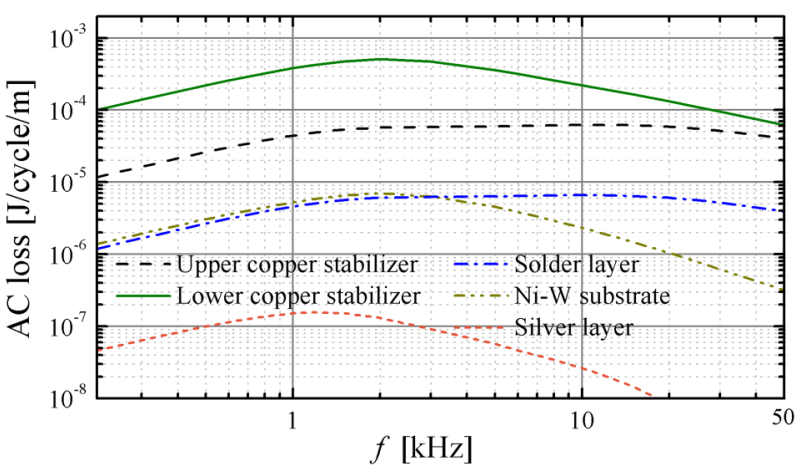

FIG. 8. FE-simulated metallic loss components (lower copper stabilizer, upper copper stabilizer, substrate, solder, and silver layer) per cycle per unit length as functions of the applied frequency with transport current $I_{p}=45 \mathrm{~A}$. 

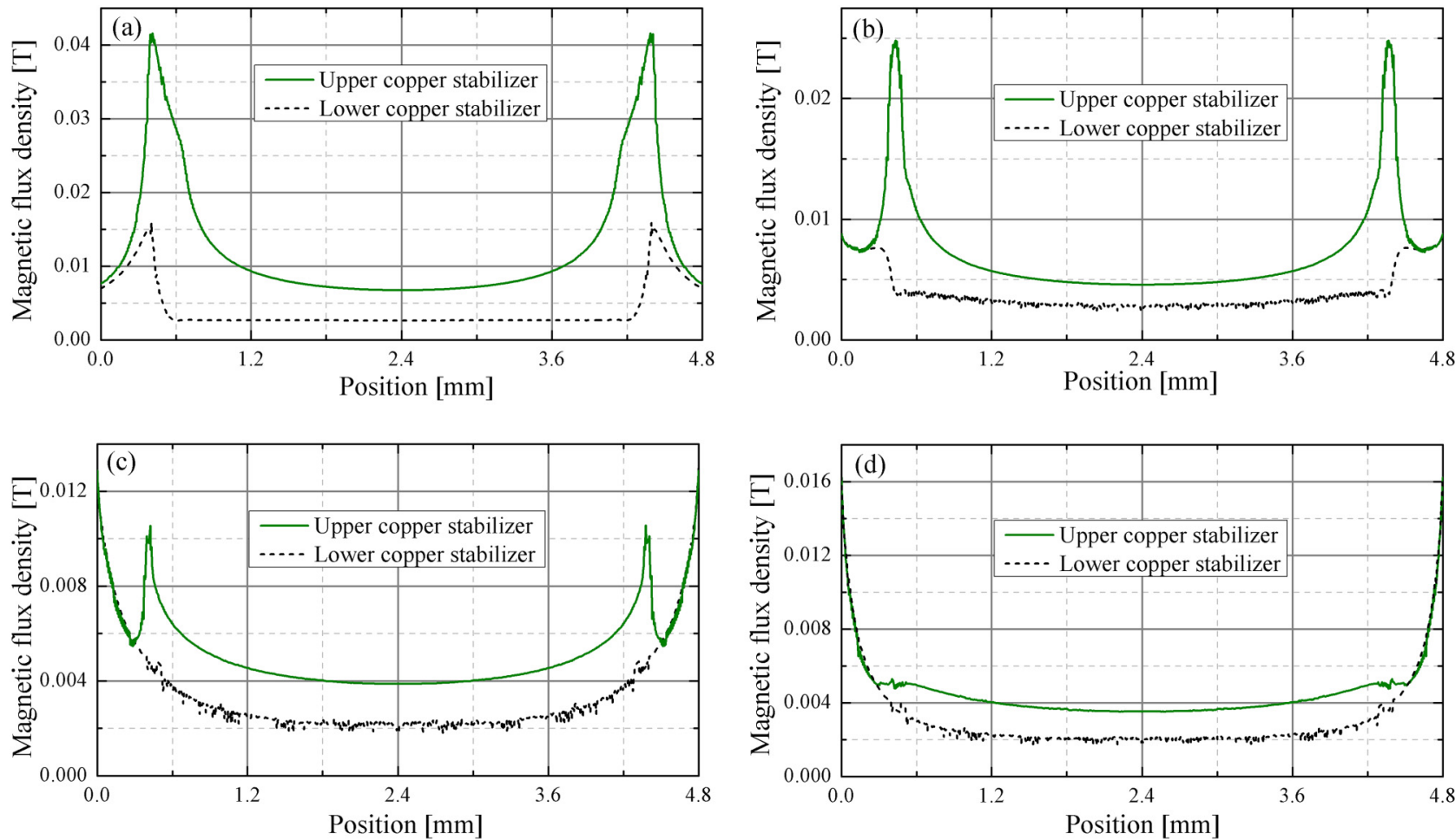

FIG. 9. FE-simulated magnetic flux density (norm) along the centerline of the two copper stabilizers at the time instant when the transport current reaches its maximum for various frequencies: (a) $500 \mathrm{~Hz}$; (b) $5 \mathrm{kHz}$; (c) $20 \mathrm{kHz}$; (d) $50 \mathrm{kHz}$. The horizontal axis varies from 0 to $4.8 \mathrm{~mm}$ (the width of the copper stabilizers) with reference to Fig. 1.

reason is that the local magnetic field at the lower copper stabilizer is weaker than that at the upper copper stabilizer. To prove this, we plot in Fig. 9 the magnetic flux density along the centerline of the two copper stabilizers. At $500 \mathrm{~Hz}$ [Fig. 9(a)], the field is indeed weaker at the lower copper stabilizer than at the upper one. This can be explained in two steps. First, from Fig. 5, we observe that at $500 \mathrm{~Hz}$, most of the current flows in the superconducting layer, so the magnetic field is generated almost solely by the supercurrent and the magnetism of the substrate. Second, from Fig. 6, we notice that the magnetic fields generated by the supercurrent and the magnetic substrate have opposite directions at the lower copper stabilizer level and the same direction at the upper copper stabilizer level. In other words, the magnetic substrate shields the magnetic field generated by the supercurrent at the lower copper stabilizer level. The resulting effect is shown in Fig. 10, where we plot the normalized current distribution in the upper and lower copper stabilizers for different frequencies. It can clearly be seen that at $500 \mathrm{~Hz}$, the current flows uniformly in the lower copper stabilizer owing to the low local magnetic field, while it flows only in the corners of the upper copper stabilizer owing to the nonuniform field. As a result, the lower copper stabilizer carries a higher current than the upper one, and, therefore, exhibits higher metallic losses.

In Fig. 8, it can be seen that the losses in the two copper stabilizers converge to the same level when the frequency increases.
This can be explained in a similar fashion as above. At high frequencies, the magnetic field is generated by the supercurrent, the metallic current, and the magnetism of the substrate (Fig. 5); the magnetization of the substrate decreases (Sec. IV E); the magnetic field and the current distribution of both copper stabilizers become similar; and the metallic losses of the two copper stabilizers converge to the same level.

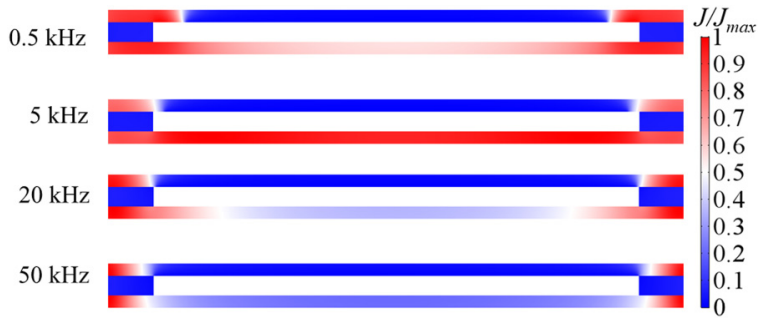

FIG. 10. FE-simulated current density (norm) distribution normalized by the maximum current density $J_{\max }$ in the copper stabilizers and the solder fillets as a function of frequency at the time instant when the transport current reaches its maximum. For the purpose of illustration, the thickness of the HTS CC has been artificially enlarged. 


\section{CONCLUSION}

We measured the frequency-dependent characteristics of the transport ac losses up to $15 \mathrm{kHz}$ in a RABiTS HTS CC with a magnetic substrate. It has been revealed that the ac losses per cycle increase with frequency up to a "transition frequency" $f_{T}$ and then decrease continuously. This feature has never been reported before, thus indicating that the present theoretical description of the CC frequency-dependent losses, established for low frequencies, is incomplete.

Through FE simulations, allowing for loss separation, it has been found that (a) the losses per cycle in the metal components become predominant at high frequency, and they increase with the frequency up to $f_{T}$, above which they start to decrease; and (b) the ferromagnetic and hysteresis losses per cycle are nearly independent of the frequency below $f_{T}$, above which they start to decrease rapidly.

From a proposed circuit model, we have argued that this can be explained by several facts: (i) when the frequency increases, part of the supercurrent starts migrating into the metallic path; (ii) the current flowing in the metallic material is not an induced eddy current as commonly thought, but rather a transport current inductively coupled to the superconducting current; (iii) the local magnetic field decreases in the substrate when the frequency increases; (iv) the critical current, which is sensitive to the local magnetic field, increases with the frequency in the presence of a magnetic substrate; and (v) the magnetic substrate shields the magnetic field generated by the supercurrent at the lower copper stabilizer level.

These findings, by supplementing the existing description of frequency-dependent losses in HTS CCs, could help in the design and optimization of new electrical applications of superconducting materials.

\section{ACKNOWLEDGMENTS}

This work was supported by the National Natural Science Foundation of China (NNSFC) under Grant No. 51722706.

\section{APPENDIX: CIRCUIT MODEL}

The circuit model (see Fig. 11) is composed of a superconducting $R L$ branch in parallel with a normal conducting metallic $R L$ branch. Similar lumped parameter models have been used for power system analysis with superconducting fault current limiters. ${ }^{59}$ Using complex notation (with an overbar), the circuit

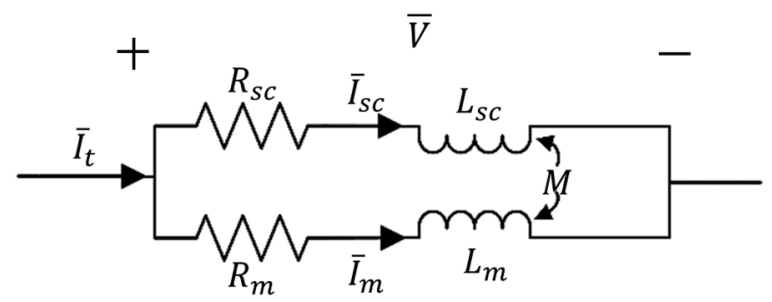

FIG. 11. Circuit model of an HTS CC. The model consists of two parallel branches: a superconducting branch and a metallic branch. equations are

$$
\begin{gathered}
\bar{V}=R_{s c} \bar{I}_{s c}+j \omega L_{s c} \bar{I}_{s c}+j \omega M \bar{I}_{m}, \\
\bar{V}=R_{m} \bar{I}_{m}+j \omega L_{m} \bar{I}_{m}+j \omega M \bar{I}_{s c}, \\
\bar{I}_{t}=\bar{I}_{s c}+\bar{I}_{m},
\end{gathered}
$$

where $\bar{V}$ is the voltage drop through the HTS CC, $\bar{I}_{t}$ is the total transport current, $\bar{I}_{s c}$ and $\bar{I}_{m}$ are the currents in the superconducting and metallic paths, respectively, $R_{s c}, R_{m}, L_{s c}$, and $L_{m}$ are the resistances and self-inductances of the superconducting and metallic paths, respectively, $M$ is the mutual inductance between the two paths, and $\omega$ is the angular frequency.

\section{REFERENCES}

${ }^{1}$ S. S. Kalsi, Applications of High Temperature Superconductors to Electric Power Equipment (IEEE Press, New Jersey, 2011).

${ }^{2}$ A. Kario, M. Vojenciak, F. Grilli, A. Kling, B. Ringsdorf, and U. Walschburger, Supercond. Sci. Technol. 26, 085019 (2013).

${ }^{3}$ W. Goldacker, F. Grilli, E. Pardo, A. Kario, S. I. Schlachter, and M. Vojenčiak, Supercond. Sci. Technol. 27, 093001 (2014).

${ }^{4}$ C. Y. Lee, J. H. Lee, J. M. Jo, C. B. Park, W. H. Ryu, Y. Do Chung, Y. J. Hwang, T. K. Ko, S. Y. Oh, and J. Lee, IEEE Trans. Appl. Supercond. 24, 3600304 (2014).

${ }^{5}$ X. Song, N. Mijatovic, J. Kellers, C. Bührer, A. V. Rebsdorf, J. Hansen, M. Christensen, J. Krause, J. Wiezoreck, H. Pütz, and J. Holbøll, IEEE Trans. Appl. Supercond. 27, 5201105 (2017).

${ }^{6} \mathrm{E}$. Pardo, M. Staines, Z. Jiang, and N. Glasson, Supercond. Sci. Technol. 28, 114008 (2015).

${ }^{7}$ N. Glasson, M. Staines, N. Allpress, M. Pannu, J. Tanchon, E. Pardo, R. Badcock, and R. Buckley, IEEE Trans. Appl. Supercond. 27, 1 (2017).

${ }^{8}$ Z. Jiang, K. Hamilton, N. Amemiya, R. A. Badcock, and C. W. Bumby, Appl. Phys. Lett. 105, 112601 (2014).

9. Geng and T. A. Coombs, Appl. Phys. Lett. 107, 142601 (2015).

${ }^{10}$ J. Geng, B. Shen, C. Li, H. Zhang, K. Matsuda, J. Li, X. Zhang, and T. A. Coombs, Appl. Phys. Lett. 108, 262601 (2016).

${ }^{11}$ R. J. Sedwick, Ann. Phys. 325, 287 (2010).

${ }^{12}$ G. Zhang, H. Yu, L. Jing, J. Li, Q. Liu, and X. Feng, IEEE Trans. Appl. Supercond. 24, 4600505 (2014).

${ }^{13}$ H. Yu, G. Zhang, L. Jing, Q. Liu, W. Yuan, Z. Liu, and X. Feng, IEEE Trans. Appl. Supercond. 25, 5000405 (2015).

${ }^{14}$ W. Wang, Y. Lei, S. Q. Huang, P. Wang, Z. Huang, and Q. Zhou, IEEE Trans. Appl. Supercond. 28, 0600804 (2018).

${ }^{15}$ R. Inoue, D. Miyagi, M. Tsuda, and H. Matsuki, IEEE Trans. Appl. Supercond. 27, 5400106 (2017)

${ }^{16}$ Y. He, Y. Wang, Y. Hu, W. Chen, and Z. Yan, Supercond. Sci. Technol. 32, 015010 (2019).

${ }^{17}$ F. Grilli, E. Pardo, A. Stenvall, D. N. Nguyen, W. J. Yuan, and F. Gömöry, IEEE Trans. Appl. Supercond. 24, 8200433 (2014).

${ }^{18}$ B. Shen, J. Li, J. Geng, L. Fu, X. Zhang, H. Zhang, C. Li, F. Grilli, and T. A. Coombs, Supercond. Sci. Technol. 30, 075006 (2017).

${ }^{19}$ M. Majoros, L. Ye, A. V. Velichko, T. A. Coombs, M. D. Sumption, and E. W. Collings, Supercond. Sci. Technol. 20, S299 (2007).

${ }^{20}$ K.-H. Müller, Physica C Supercond. 281, 1 (1997).

${ }^{21}$ G. Liu, G. Zhang, H. Yu, L. Jing, L. Ai, and Q. Liu, J. Appl. Phys. 121, 243902 (2017).

${ }^{22}$ F. Gömöry, E. Janíková, and J. Šouc, Supercond. Sci. Technol. 15, 1345 (2002).

${ }^{23}$ R. Inoue, D. Miyagi, M. Tsuda, and H. Matsuki, IEEE Trans. Appl. Supercond. 27, 4702005 (2017). 
${ }^{24}$ G. Li, H. Liu, Y. Wang, and H. Zhang, IEEE Trans. Appl. Supercond. 26, 8201004 (2016).

${ }^{\mathbf{2 5}}$ M. Polak, J. Kvitkovic, P. Mozola, E. Usak, P. N. Barnes, and G. A. Levin, Supercond. Sci. Technol. 20, S293 (2007).

${ }^{26}$ J. Yuan, J. Fang, P. Qu, G. X. Shen, and Z.-H. Han, Physica C Supercond. 424, 72 (2005).

${ }^{27}$ M. Sander and F. Grilli, J. Phys. Conf. Ser. 234, 022030 (2010).

${ }^{28}$ K. P. Thakur, A. Raj, E. H. Brandt, J. Kvitkovic, and S. V. Pamidi, Supercond. Sci. Technol. 24, 065024 (2011).

${ }^{29}$ G. Liu, G. Zhang, L. Jing, H. Yu, L. Ai, W. Yuan, and W. Li, IEEE Trans. Appl. Supercond. 27, 6603807 (2017).

${ }^{30}$ D. N. Nguyen, P. V. P. S. S. Sastry, and J. Schwartz, J. Appl. Phys. 101, 053905 (2007).

${ }^{31}$ S. Li, D.-X. Chen, and J. Fang, Supercond. Sci. Technol. 28, 125011 (2015).

${ }^{32}$ F. Gömöry, M. Vojenčiak, E. Pardo, M. Solovyov, and J. Šouc, Supercond. Sci. Technol. 23, 034012 (2010).

${ }^{33}$ D. Miyagi, Y. Amadutsumi, N. Takahashi, and O. Tsukamoto, IEEE Trans. Appl. Supercond. 17, 3167 (2007).

${ }^{34}$ M. D. Ainslie, T. J. Flack, and A. M. Campbell, Physica C Supercond 472, 50 (2012).

${ }^{35}$ D.-X. Chen, E. Pardo, and A. Sanchez, Supercond. Sci. Technol. 17, 417 (2004).

${ }^{36}$ C. M. Friendt, C. Beduzf, B. Dutoit, R. Navarro, E. Ceredap, and J. Alonso-llorente, IEEE Trans. Appl. Supercond. 9, 1165 (1999).

${ }^{37}$ N. Nibbio, S. Stavrev, and B. Dutoit, Physica C Supercond. 310, 208 (1998).

${ }^{38}$ T. Fukunaga and A. Oota, Physica C Supercond. 251, 325 (1995).

${ }^{39}$ S. Stavrev and B. Dutoit, Physica C Supercond. 310, 86 (1998).

${ }^{40}$ B. Y. Shen, J. Li, J. Z. Geng, L. Fu, X. C. Zhang, C. Li, H. Zhang, Q. H. Dong, J. Ma, and T. A. Coombs, Physica C Supercond. 541, 40 (2017).

${ }^{41}$ W. T. Norris, J. Phys. D 3, 489 (1970).

${ }^{42}$ A. M. Campbell, IEEE Trans. Appl. Supercond. 5, 682 (1995).

${ }^{43}$ M. Ciszek, S. P. Ashworth, B. A. Glowacki, A. M. Campbell, and P. Haldar,

Physica C Supercond. 272, 319 (1996).
${ }^{44}$ Y. Yang, T. Hughes, C. Beduz, D. M. Spiller, R. G. Scurlock, and W. T. Norris, Physica C Supercond. 256, 378 (1996).

${ }^{45}$ O. Tsukamoto, J. Ogawa, M. Ciszek, D. Miyagi, I. Okazaki, Y. Niidome, and S. Fukui, IEEE Trans. Appl. Supercond. 11, 2208 (2001).

${ }^{46}$ S. Fukui, Y. Kitoh, T. Numata, O. Tsukamoto, J. Fujikami, and K. Hayashi, Advances in Cryogenic Engineering (Springer, 1998), Vol. 44, p. 723.

${ }^{47}$ J. J. Rabbers, B. Ten Haken, and H. H. J. T. Kate, Rev. Sci. Instrum. 72, 2365 (2001).

${ }^{48}$ Z. Hong, A. M. Campbell, and T. A. Coombs, Supercond. Sci. Technol. 19, 1246 (2006).

${ }^{49}$ D. N. Nguyen, S. P. Ashworth, J. O. Willis, F. Sirois, and F. Grilli, Supercond. Sci. Technol. 23, 025001 (2010).

${ }^{50}$ M. Zhang, J. Kvitkovic, J. H. Kim, C. H. Kim, S. V. Pamidi, and T. A. Coombs, Appl. Phys. Lett. 101, 102602 (2012).

${ }^{51}$ L. Quéval, V. M. R. Zermeño, and F. Grilli, Supercond. Sci. Technol. 29, 024007 (2016).

${ }^{52}$ V. M. R. Zermeño, A. B. Abrahamsen, N. Mijatovic, B. B. Jensen, and M. P. Sørensen, J. Appl. Phys. 114, 173901 (2013).

${ }^{53}$ R. C. Weast, CRC Handbook of Chemistry and Physics (CRC Press, Inc., 2001).

${ }^{54}$ K. Nam, C. Lee, D. K. Park, T. K. Ko, and B. Y. Seok, IEEE Trans. Appl. Supercond. 17, 1923 (2007).

${ }^{55}$ D. Miyagi, Y. Yunoki, M. Umabuchi, N. Takahashi, and O. Tsukamoto, Physica C 468, 1743 (2008).

${ }^{56}$ D. N. Nguyen, S. P. Ashworth, and J. O. Willis, J. Appl. Phys. 106, 093913 (2009).

${ }^{57}$ R. C. Duckworth, J. R. Thompson, M. J. Gouge, J. W. Lue, A. O. Ijaduola, D. Yu, and D. T. Verebelyi, Supercond. Sci. Technol. 16, 1294 (2003).

${ }^{58} \mathrm{~N}$. Amemiya, Z. Jiang, Z. Li, M. Nakahata, T. Kato, M. Ueyama, N. Kashima, S. Nagaya, and S. Shiohara, Physica C Supercond. 468, 1718 (2008).

${ }^{59}$ J. Duron, F. Grill, B. Dutoit, and S. Svetlomir, Physica C 401, 231 (2004). 